03

\title{
Моделирование удара вихревого кольца о твердую поверхность
}

\author{
() П.А. Куйбин, С.Г. Скрипкин, М.А. Цой, С.И. Шторк \\ Институт теплофизики им. С.С. Кутателадзе СО РАН, Новосибирск, Россия \\ E-mail: kuibin@itp.nsc.ru
}

Поступило в Редакцию 14 сентября 2018 г.

\begin{abstract}
Проведено экспериментальное и теоретическое исследование эффекта взаимодействия вихревого кольца с твердой поверхностью. Приводятся аналитические формулы, позволяющие описать импульсы давления, возникающие при прохождении вихревого кольца вблизи плоской поверхности. Сопоставлены формы импульсов давления с зарегистрированными в эксперименте. Полученные результаты могут быть использованы для прогнозирования нерасчетных режимов работы гидротурбины, характеризующихся апериодическими сильными ударами в гидротурбинном тракте за рабочим колесом.
\end{abstract}

DOI: 10.21883/PJTF.2019.01.47155.17527

Режимы работы гидротурбины трудно прогнозируемы по причине очень сложной гидродинамической структуры течения. В частности, в потоке за рабочим колесом могут развиваться неустойчивости или отрыв потока, что приводит к опасным вибрациям турбины. Наиболее сильные колебания регистрируются в гидротурбинах типа Фрэнсиса, работающих в неоптимальных условиях. Одним из основных источников колебаний является так называемый „вихревой жгут“ вихрь винтовой формы, образующийся за рабочим колесом в конической части отсасывающей трубы. Регулярные периодические типы колебаний хорошо изучены (см. обзоры в $[1,2])$. Недавние исследования $[3,4]$ структуры потока за моделью рабочего колеса показали, что при определенных режимах работы гидротурбины винтовой вихревой жгут может быть неустойчивым. С помощью высокоскоростной камеры было обнаружено [3], что иногда соседние витки спирали сближаются друг с другом и возникает явление перезамыкания с дальнейшей генерацией вихревого кольца. Два сценария перезамыкания были выявлены в экспериментах. В одном случае наблюдалось вихревое кольцо, связанное с главным винтообразным вихрем. В другом случае было обнаружено, что вихревое кольцо отделяется от основного вихря, после чего по спиральной траектории приближается к стенке канала. В экспериментах с синхронизацией высокоскоростной съемки с измерениями давления на стенке канала [5] были зафиксированы сильные импульсы давления в тот момент, когда вихревое кольцо проходило вблизи зонда. События перезамыкания, а также импульсы давления, вызванные отделившимся вихревым кольцом, имеют непериодический характер. Такие явления относятся к случайным (или апериодическим) пульсациям, представляющим особый интерес из-за того, что их природа намного сложнее, чем в случае регулярных пульсаций, а спрогнозировать их практически невозможно. Недавно [6] была разработана физико-математическая модель для описания импульсов давления, возникающих при прохождении вихревого кольца вблизи плоской поверхности. В настоящей ра- боте приводится анализ возможных форм импульсов в зависимости от параметров вихря, его положения относительно поверхности и траектории движения вихря. Проводится сопоставление с различными формами импульсов, зарегистрированными в эксперименте.

Вихревое кольцо, наблюдаемое в упомянутых выше экспериментах, имело довольно малый размер относительно диаметра канала. Это позволяет, как и в работе [6], рассмотреть задачу в упрощенной постановке: заменить коническую поверхность на плоскую. Кроме того, мы используем невязкую аппроксимацию, пренебрегая поверхностным пограничным слоем и диффузией вихря. Пусть вихревое кольцо имеет интенсивность $Г$, радиус $a$, угол наклона к поверхности $\alpha$ с минимальным расстоянием $d$ от поверхности и центр, расположенный в точке $\left(x_{0}, y_{0}, z_{0}\right)$. Плоскость $y=0$ является плоскостью симметрии. Влияние поверхности на поле скоростей можно учесть путем введения зеркально отраженного воображаемого вихревого кольца с противоположной интенсивностью -Г. Последнее предположение о равномерном распределении завихренности внутри вихревого ядра радиуса $r_{c}$ и потенциальном течении вне ядра - позволяет применить известные аналитические подходы (см., например, [7]) для нахождения распределения давления на плоской поверхности.

Действительно, применяя закон Био-Савара к круговой вихревой нити, можно найти индуцированное поле скоростей

$$
\begin{gathered}
u_{\rho}(\rho, \xi)=\frac{\Gamma}{8 \pi \rho} \frac{\zeta}{(\rho a)^{1 / 2}} k\left[\frac{2-k^{2}}{1-k^{2}} E(k)-2 K(k)\right], \\
u_{\zeta}(\rho, \zeta)=\frac{\Gamma}{8 \pi \rho}\left(\frac{a}{\rho}\right)^{1 / 2} k\left[\frac{k^{2}}{1-k^{2}} E(k)\right. \\
\left.-\frac{\rho}{a}\left(\frac{2-k^{2}}{1-k^{2}} E(k)-2 K(k)\right)\right]
\end{gathered}
$$

где $K(k)$ и $E(k)$ - полные эллиптические интегралы первого и второго типа, $k^{2}=4 \rho a /\left[(\rho+a)^{2}+\xi^{2}\right]$; локальные цилиндрические координаты $(\rho, \theta, \xi)$ связаны с 
декартовыми координатами $(x, y, z)$ следующими соотношениями:

$$
\begin{gathered}
\rho^{2}=\left[\left(x-x_{0}\right) \cos \alpha+\left(z-z_{0}\right) \sin \alpha\right]^{2}+y^{2}, \\
\tan \theta=y /\left[\left(x-x_{0}\right) \cos \alpha+\left(z-z_{0}\right) \sin \alpha\right], \\
\zeta=\left(z-z_{0}\right) \cos \alpha-\left(x-x_{0}\right) \sin \alpha ; \\
x=\rho \cos \theta \cos \alpha-\zeta \sin \alpha+x_{0}, \quad y=\rho \sin \theta, \\
z=\rho \cos \theta \sin \alpha+\zeta \cos \alpha+z_{0} .
\end{gathered}
$$

Компоненты скорости в декартовых координатах также могут быть выражены через значения скорости в цилиндрической системе координат (1)

$$
\begin{gathered}
u_{x}=u_{\rho} \cos \theta \cos \alpha-u_{\xi} \sin \alpha, \quad u_{y}=u_{\rho} \sin \theta, \\
u_{z}=u_{\rho} \cos \theta \sin \alpha+u_{\xi} \cos \alpha .
\end{gathered}
$$

Для определения давления $\rho$ применяется интеграл Коши-Лагранжа

$$
\frac{\partial \varphi}{\partial t}+\frac{\mathbf{u}^{2}}{2}+\frac{p}{\rho_{l}}=\text { const. }
$$

Здесь и - вектор скорости, $\rho_{l}-$ плотность жидкости, $\varphi$ - потенциал скорости, который может быть представлен в виде интеграла

$$
\begin{aligned}
& \varphi=\frac{\Gamma}{4 \pi} a \xi \\
& \times \int_{0}^{2 \pi} \frac{a-\rho \cos \beta}{\left[\rho^{2}+a^{2}-2 \rho a \cos \beta\right]\left[\zeta^{2}+\rho^{2}+a^{2}-2 \rho a \cos \beta\right]^{1 / 2}} d \beta .
\end{aligned}
$$

В дополнение к (5) может существовать потенциальное течение, не связанное с вихревым кольцом, например однородный поток вдоль поверхности в произвольном направлении. Потенциал (5) является функцией следующих переменных и параметров: $\varphi\left(x, y, z ; \Gamma, a, \alpha, x_{0}, z_{0}\right)$. Очевидно, что $\partial \varphi / \partial \rho=u_{\rho}, \partial \varphi / \partial \xi=u_{\xi}$, так же как $\partial \varphi / \partial x=u_{x}, \partial \varphi / \partial y=u_{y}, \partial \varphi / \partial z=u_{z}$. Как видно, потенциал явно не зависит от времени. Следовательно,

$$
\frac{\partial \varphi}{\partial t}=\frac{\partial \varphi}{\partial a} \frac{d a}{d t}+\frac{\partial \varphi}{\partial \alpha} \frac{d \alpha}{d t}+\frac{\partial \varphi}{\partial x_{0}} \frac{d x_{0}}{d t}+\frac{\partial \varphi}{\partial z_{0}} \frac{d z_{0}}{d t} .
$$

Параметр $\Gamma$ - интенсивность вихря - является константой. Формулы (1), (5) описывают скорость $\mathbf{u}^{r}$ и потенциал $\varphi^{r}$, индуцируемые вихревым кольцом в безграничном пространстве. При наличии плоской поверхности к ним добавляются вклады $\mathbf{u}^{i}$ и $\varphi^{i}$, индуцируемые отраженным (мнимым) вихревым кольцом. В результате, как показано в [6], можно найти выражения для трех частных производных из правой части формулы (6)

$$
\begin{gathered}
\frac{\partial \varphi}{\partial x_{0}}=-u_{x}^{i}-u_{x}^{r}, \quad \frac{\partial \varphi}{\partial z_{0}}=u_{z}^{i}-u_{z}^{r}, \\
\frac{\partial \varphi}{\partial \alpha}=u_{x}^{r}\left(z-z_{0}\right)-u_{x}^{i}\left(z+z_{0}\right)-\left(u_{z}^{r}-u_{z}^{i}\right)\left(x-x_{0}\right) .
\end{gathered}
$$

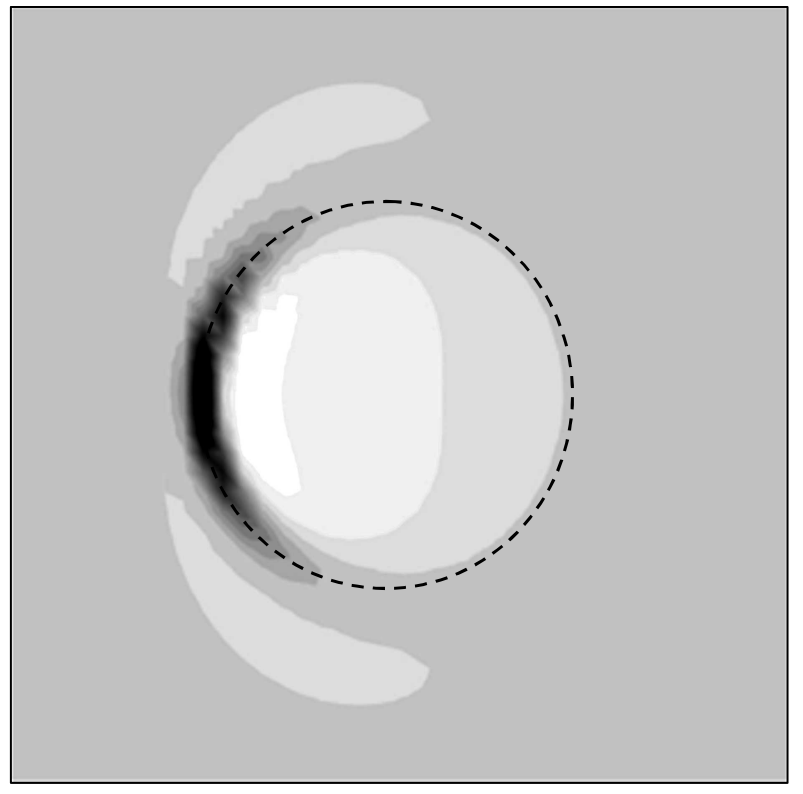

Рис. 1. Распределение давления на плоскости под вихревым кольцом. $d / a=0.1, r_{c} / a=0.1, \alpha=15^{\circ}$.

Производная $\partial \varphi / \partial а$ представлена в [6] через функции $K$ и $E$. Поскольку требуется найти распределение давления на плоскости $z=0$, выражения для частных производных упрощаются: $\partial \varphi / \partial x_{0}=-u_{x}, \partial \varphi / \partial z_{0}=0$, $\partial \varphi / \partial \alpha=-z_{0}\left(u_{x}^{r}+u_{x}^{i}\right)$,

$$
\frac{\partial \varphi}{\partial a}=-\frac{\Gamma}{4 \pi} \frac{\xi}{\rho} \frac{1}{(a \rho)^{1 / 2}} \frac{k^{3}}{1-k^{2}} E(k) .
$$

Далее необходимо найти скорость изменения параметров вихревого кольца. В первую очередь рассмотрим скорость движения его центра. Каждая точка вихревого кольца движется со скоростью, индуцируемой отраженным кольцом, плюс самоиндуцированная скорость [7]:

$$
\mathbf{u}^{s}=\frac{\Gamma}{4 \pi a}\left[\ln \left(\frac{8 a}{r_{c}}\right)-\frac{1}{4}\right]\left(\begin{array}{c}
-\sin \alpha \\
0 \\
\cos \alpha
\end{array}\right) .
$$

Для оценки скорости изменения радиуса вихревого кольца и угла его наклона относительно плоскости в [6] проведена дискретизация: разбиение кольца на $N$ одинаковых дуг и расчет положения центров дуг через интервал времени $\Delta t$. Установлено, что и изменение радиуса кольца, и наклон при малых интервалах времени линейно зависят от $\Delta t$, что позволяет найти их предельные значения при $\Delta t \rightarrow 0$.

Пример расчета распределения давления при $d / a=$ $=0.1, r_{c} / a=0.1$ и $\alpha=15^{\circ}$ представлен на рис. 1. Данные приводятся в безразмерном виде, где за масштаб длины взят радиус вихревого кольца, а в качестве масштаба скорости выбран множитель из формулы для самоиндуцированной скорости $\Gamma /(4 \pi a)$. Проекция вихревого кольца на плоскость $(x, y)$ показана штриховой 

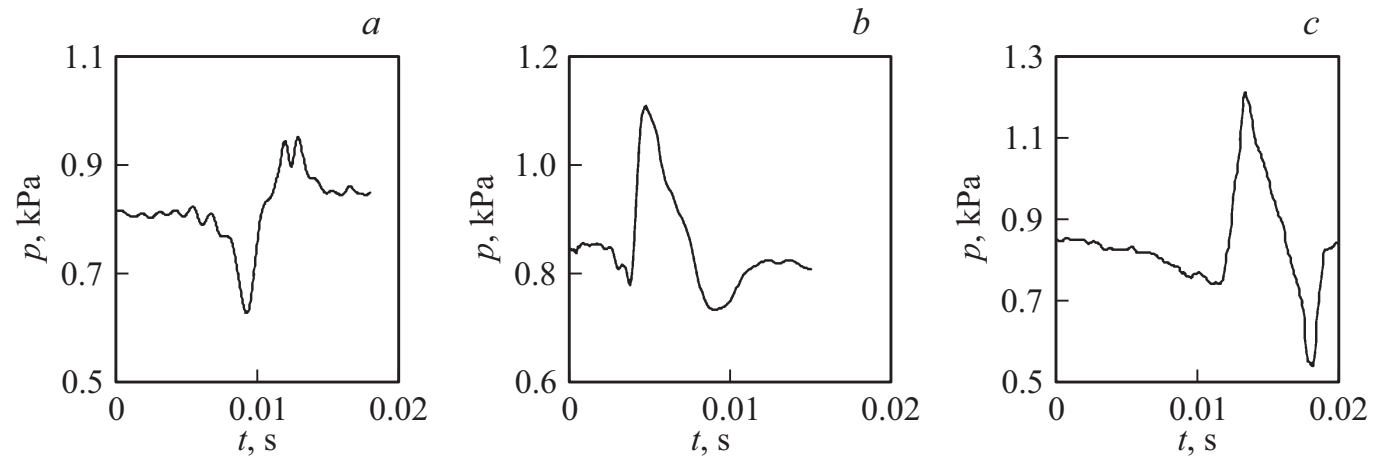

Рис. 2. Характерные формы импульсов давления, наведенных вихревыми кольцами. Пояснение в тексте.
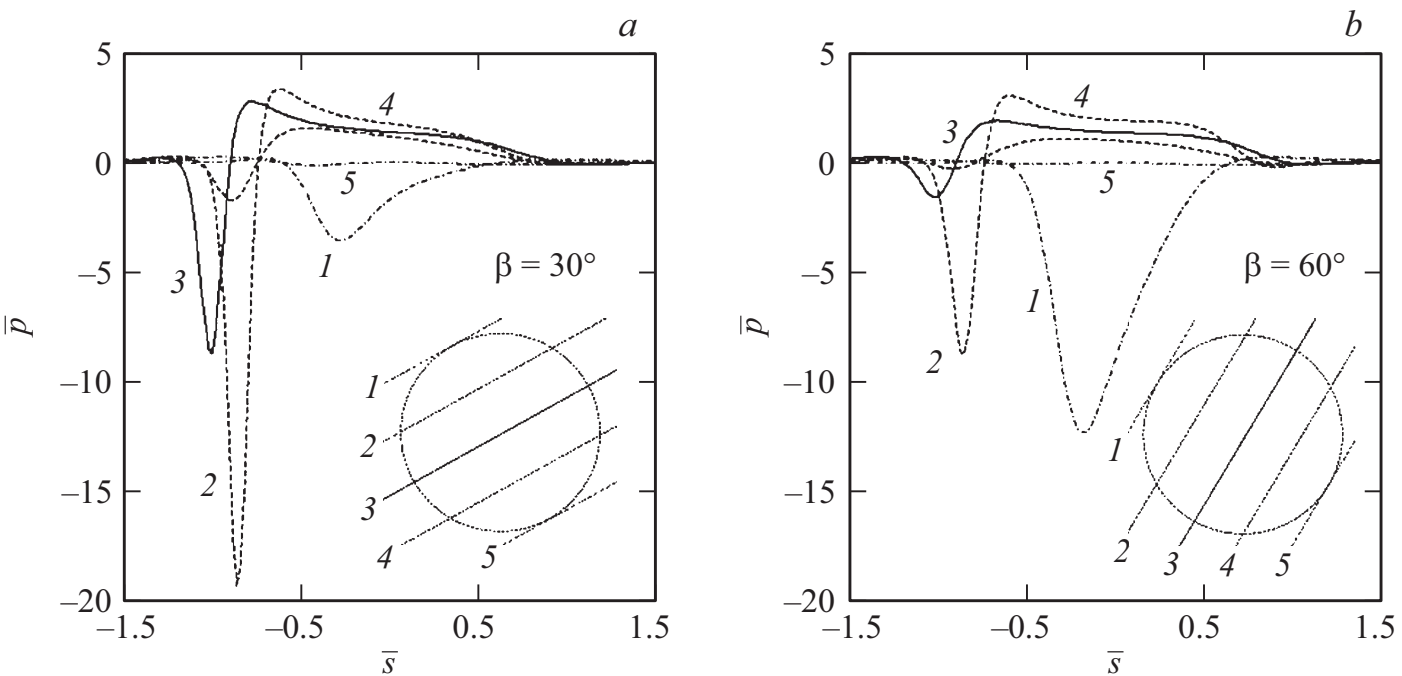

Рис. 3. Профили давления для различных траекторий (показаны на вставках) движения вихревого кольца относительно точки наблюдения. Пояснение в тексте.

линией. Минимум давления расположен под участком кольца, расположенным ближе всего к поверхности. Имеется и максимум давления, расположенный достаточно близко к минимуму.

Эксперименты по изучению процесса перезамыкания между участками винтообразного вихря с последующим формированием вихревых колец и их взаимодействием со стенкой канала проводились на экспериментальном стенде в Институте теплофизики им. С.С. Кутателадзе CO РАН, в лаборатории экологических проблем теплоэнергетики. Стенд представляет собой замкнутый гидродинамический контур с рабочим участком, моделирующим конический участок отсасывающей трубы гидротурбины. Вместо рабочего колеса используется комбинация стационарного и свободно вращающегося завихрителей, позволяющих получать распределение скорости на входе в отсасывающую трубу, подобное натурным [5]. В экспериментах проводилась высокоскоростная визуализация течения с использованием камеры CMOS PCO 1200.hs с частотой съемки $500 \mathrm{fps}$. Видеосъемка была синхронизирована с регистрацией давления пьезорезистивным датчиком Keller PA-4LC для одновременной записи кар- тины течения и реализаций сигнала датчика давления. Анализ данных позволил установить однозначную связь между отрывами вихревых колец и резкими импульсами давления на стенке конуса.

Характерные формы импульсов давления, связанных с прохождением вихревого кольца вблизи стенки, представлены на рис. 2. В одних случаях возникает разрежение с последующим максимумом давления, в других сначала скачок давления с последующим минимумом меньшей амплитуды. Наблюдались и импульсы примерно одинакового роста давления и последующего падения.

Предположим, что скорость трансляционного (потенциального) движения вихря значительно выше скорости самоиндуцированного движения и других индуцированных эффектов. В этом случае форма временно́го импульса в приведенной выше модели будет зависеть от угла наклона вихревого кольца и траектории его движения. В системе координат, связанной с вихрем, точка наблюдения (точка регистрации давления в эксперименте) будет двигаться вдоль некоторой линии, пересекающей плоскость изображения на рис. 1. Распределения давления вдоль таких линий (или траекторий) 
и будем сравнивать с экспериментальными данными. Как видно из рис. 3, в модели наиболее вероятная форма импульса соответствует импульсу, показанному на рис. 2, $a$ (сильное падение давления с последующим относительно небольшим максимумом). Порядок следования минимумов и максимумов давления может меняться в зависимости от знака угла наклона кольца и направления его движения.

Положительный импульс с последующим минимумом давления (рис. $2, b$ ) возможен, если траектория наклонена под большим углом (например, $\beta=60^{\circ}$ ) и лежит ниже центра кольца (штриховые линии на рис. $3, b$ ). Формы импульса с положительными и отрицательными пиками близкой амплитуды (рис. 2,c) могут возникать при умеренном наклоне траектории (штриховые линии на рис. $3, a)$. Положительные пики на рис. 3 имеют большую ширину (расчет проведен для $\alpha=15^{\circ}$ ); для более высоких углов наклона кольца они будут более узкими.

В данной работе упрощенная модель вихревого кольца применена для анализа зарегистрированных в экспериментах импульсов давления при прохождении вихревых колец вблизи твердой поверхности. В модели используются теоретические подходы, позволяющие описать в аналитическом виде поле скорости и потенциал, индуцированный вихревым кольцом, и как следствие вывести зависимости для распределения давления, наведенного вихревым кольцом, наклоненным к плоскости под некоторым углом. Проведенное сопоставление модельных распределений давления с данными экспериментов позволяет сделать вывод об адекватности разработанной модели. Результаты работы могут быть использованы для прогнозирования нерасчетных режимов работы гидротурбины, характеризующихся апериодическими сильными ударами за рабочим колесом.

Работа выполнена при поддержке Российского научного фонда (грант № 16-19-00138).

\section{Список литературы}

[1] Nicolet $C$. Hydroacoustic modelling and numerical simulation of unsteady operation of hydroelectric systems. PhD thesis. Lausanne: EPFL, 2007. 314 p.

[2] Dörfler P., Sick M., Coutu A. Flow-induced pulsation and vibration in hydroelectric machinery. London: Springer-Verlag, 2013. 242 p. DOI: $10.1007 / 978-1-4471-4252-2$

[3] Алексеенко С.В., Куйбин П.А., Шторк С.И., Скрипкин С.Г., Цой М.А. // Письма в ЖЭТФ. 2016. Т. 103. В. 7. С. 516-521. DOI: $10.7868 / \mathrm{S} 0370274 \mathrm{X} 16070067$

[4] Платонов Д.В., Минаков А.В., Дектерев Д.А., Масленникова А.В., Куйбин П.А. // Письма в ЖТФ. 2017. Т. 43. В. 21. C. 33-39. DOI: 10.21883/PJTF.2019.01.47155.17527

[5] Skripkin S.G., Tsoy M.A., Kuibin P.A., Shtork S.I. // J. Fluids Eng. 2017. V. 139. P. 081103 (1-7). DOI: 10.1115/1.4036264

[6] Kuibin P.A. // J. Phys.: Conf. Ser. 2017. V. 891. P. 012082 (1-6). DOI:10.1088/1742-6596/891/1/012082

[7] Ламб Г. Гидродинамика. М.-Л.: Гостехиздат, 1947. 928 с. 